\title{
CNAP - EVOLUTION OF CONTINUOUS NON-INVASIVE ARTERIAL BLOOD PRESSURE MONITORING
}

\author{
Fortin $\mathrm{J}^{1}$, Wellisch $\mathrm{A}^{1}$, Maier $\mathrm{K}^{1}$ \\ ${ }^{1}$ CNSystems Medizintechnik AG, Reininghausstrasse 13, 8020 Graz, Austria \\ juergen.fortin@cnsystems.at
}

\begin{abstract}
Blood pressure (BP) is one of the most important cardiovascular parameters in nearly every care area. $A$ method for continuous non-invasive arterial pressure (CNAP) monitoring is described. CNAP is especially designed for perioperative, critical and emergency care. It concentrates on the clinically important BPinformation such as absolute BP and BP-changes as well as physiological BP-rhythms and BP-waveforms, where other hemodynamic parameters like cardiac output (CO) and dynamic fluid management parameters (e.g. pulse pressure variation $P P V)$ can be derived. For this kind of hemodynamic information, tailor-made mechanisms have been developed. Recent validation studies underline accuracy and clinical acceptance of the CNAP technology.
\end{abstract}

Keywords: Continuous, non-invasive, blood pressure

\section{Introduction}

Up to now beat-to-beat BP monitoring has required invasive placement of an intra-arterial catheter (IBP). Due its invasive nature, IBP is applied in less than $20 \%$ of surgical patients [1]. The remaining $80 \%$ are monitored with intermittent upper arm sphygmomanometers (NBP), although discontinuous NBP is unable to track hemodynamic instabilities in up to $40 \%$ of all cases [2].

In addition, the concept of hemodynamic optimization using continuous BP and its derived parameters such as $\mathrm{CO}, \mathrm{PPV}$, etc. has shown an increase in medical outcome and thus has rapidly found acceptance in anesthesia and critical care [3]. Hemodynamic optimization could be performed in about $30 \%$ of all surgical procedures, if noninvasive continuous BP information would be available [1]. Non-invasive BP monitoring with accurate BP tracing as well as high-fidelity waveform to obtain hemodynamic parameters would be of high clinical interest.

However, patients in the OR, ICU or ER often receive high dose rates of vasoactive drugs to get their hemodynamic status regulated. These drugs not only induce fast changes in BP but also change the vascular tone of the peripheral arteries. Such vasomotor changes have a significant impact on the accuracy of BP in non-invasive technologies. Thus, older technologies have struggled with providing accurate values due to their lack of vasomotor artefact rejection $[4,5,6]$. CNAP was specifically designed to overcome these limitations. In this article the technological principles of CNAP and its advantages are briefly described and validation data is shown.

\section{Methods \\ Continuous BP is measured with the help of a finger sen- sor combining two methods: The "Vascular Unloading Technique" (VUT) [7] is used for BP-rhythms and BP- waveforms and the new VERIFI-technology controls periods of slower BP-changes, where vasomotor tone may influence the measurement. Further, the resulting BP- signal is scaled to an initial upper arm NBP (absolute BP) by means of a mathematical transfer function (Figure 1).}

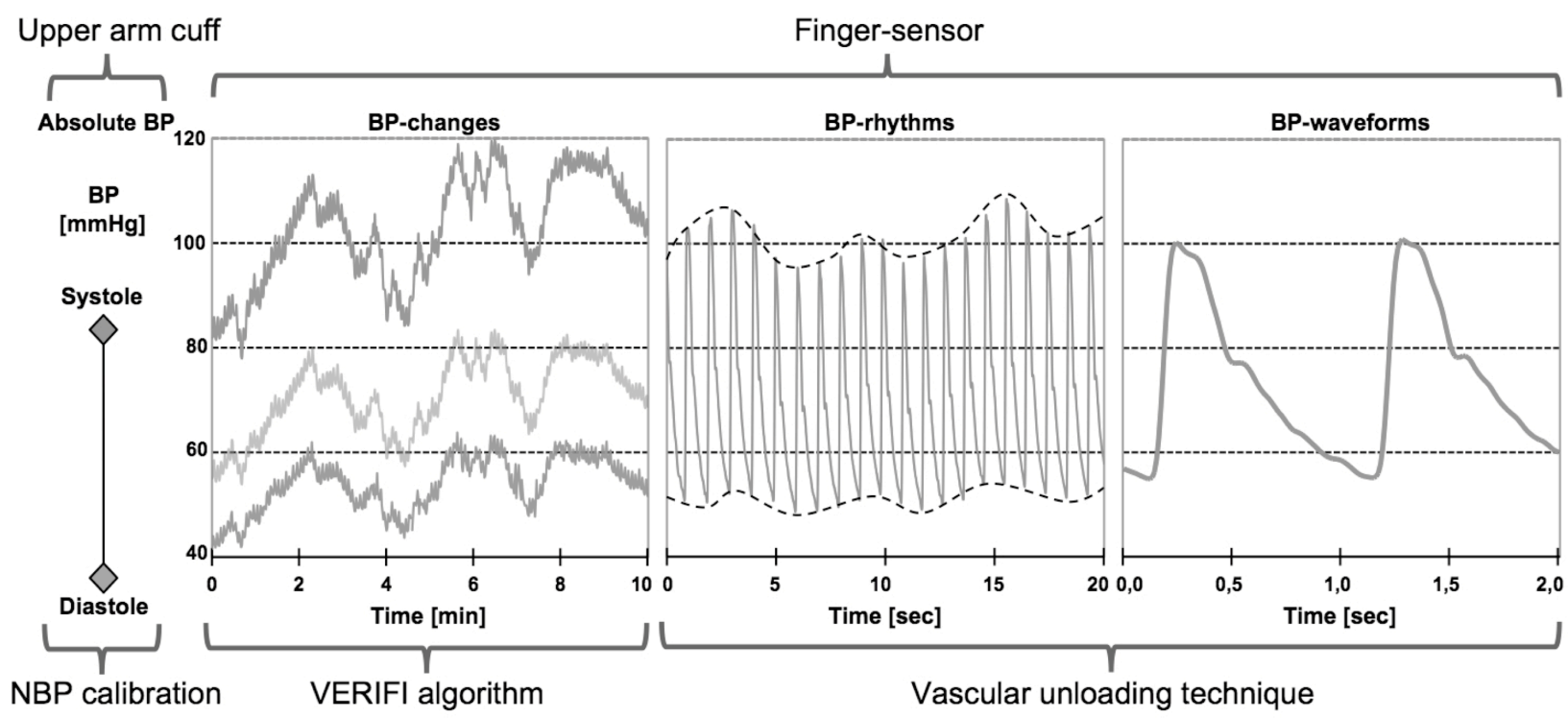

Figure 1: Interaction between the measurement locations (upper arm cuff and finger sensor) and technological methods (NBP-calibration, VERIFI and VUT) used for different BP-information. 
Upper arm cuff BP is the gold standard in clinical practice [8]. Due to its daily use in clinical routine it is obvious that the physician wants to obtain continuous BP that is as accurate as the gold standard. Thus, a mathematical calibration to NBP is performed using a transfer function [9]. VUT is a sound method for measuring BP rhythms and waveforms. Typical VUT methods use a single control loop [10], which has to deal with fast pressure increase and release in the cuff as well as with the tracking of BP changes for the stability of the system. In contrast to older methods, a number of interlocking control loops is used within CNAP. Each inner loop is responsible for a welldefined characteristic of its underlying control mechanism and provides near-ideal conditions for the surrounding outer control loops. There are multiple loops for BPwaveforms and a loop for slower BP-rhythms [11].

However, for BP-changes and long-term stability VUT is directly affected by changes in vasomotor tone. Thus, for the measurement of BP-changes, the so-called VERIFIalgorithm has been designed:

Vasomotor changes typically affect the long-term BP changes in a time range below physiological BP-rhythms. This time range is filtered out and the VUT system operates with the signal content of BP-rhythms and pulse waves only. Thus, the vasomotor effect is eliminated, but also all relevant information of BP-changes. For the restoration, the VERIFI-algorithm inspects the waveforms on a beat-per-beat basis and identifies, if the control loop set point corresponds to mean BP. When there is a deviation, the system corrects the set point on an adaptive basis until the set point equals mean BP [9]. This "Vasomotoric Elimination and Reconstructed IdentiFication of the Initial set point" names the VERIFI-algorithm.

The accuracy and clinical value of CNAP was tested in several international clinical trials shown in Tab. 1 and 2 .

\section{Results}

Table 1: Differences of IBP and CNAP (BP-changes):

\begin{tabular}{l|c|c|c|c}
\hline $\begin{array}{l}\text { Study } \\
\text { (First Author) }\end{array}$ & $\begin{array}{c}\text { Care } \\
\text { Area }\end{array}$ & $\begin{array}{c}\text { Pa- } \\
\text { tients }\end{array}$ & $\begin{array}{c}\text { Bias } \\
\mathrm{mmHg}\end{array}$ & $\begin{array}{c}\text { SD } \\
\mathrm{mmHg}\end{array}$ \\
\hline Jagadeesh, 2012 [12] & ICU & 30 & $-0,04$ & 2,05 \\
Neuner, 2012 [13] & OR & 100 & $-3,1$ & 9,45 \\
Bias 2012 [14] & OR & 25 & $-1,8$ & 10,3 \\
Ilies, 2012 [15] & OR & 90 & $-4,3$ & 10,4 \\
Jeleazcov, 2010 [16] & OR & 88 & $-1,6$ & 11 \\
Monnet, 2012 [17] & ICU & 47 & 5 & 11 \\
\hline
\end{tabular}

Table 2: Validation of BP-rhythms (PPV):

\begin{tabular}{l|c|c|c|c}
\hline $\begin{array}{l}\text { Study } \\
\text { (First Author) }\end{array}$ & $\begin{array}{c}\text { Care } \\
\text { Area }\end{array}$ & $\begin{array}{c}\text { Pa- } \\
\text { tients }\end{array}$ & $\begin{array}{c}\text { Bias } \\
\%\end{array}$ & $\begin{array}{c}\text { SD } \\
\%\end{array}$ \\
\hline Monnet, 2012 [17] & ICU & 47 & $-0,6$ & 2,3 \\
Bias 2011 [18] & OR & 35 & 0,6 & 2,6 \\
\hline
\end{tabular}

\section{Discussion}

Studies in Tab. 1 show that CNAP provides BP traces in OR and ICU during daily routine within a clinically acceptable difference to IBP. PPV can accurately be derived from the CNAP-signal (Tab. 2). Further ongoing research shows accurate CO-estimation from pulse wave analysis (not shown). This high fidelity BP has technically become possible by combining VUT, NBP calibration and the VERIFI algorithm. The demonstrated accuracy, ease of use and non-invasive nature of the new CNAP technology allows clinicians to rapidly obtain all relevant BP information. CNAP allows for a new standard in routine patient monitoring in such areas where continuous hemodynamic information can clearly increase patient outcome.

\section{Acknowledgement}

The authors are employees of CNSystems Medizintechnik AG, which has developed and markets CNAP products.

\section{Bibliography}

[1] S. Maguire et al.: Respiratory Variation in Pulse Pressure and Plethysmographic Waveforms: Intrapoperative Applicability in a American Academic Center. Anesth Analg. 2011; 112(1): 94-96

[2] C. Ilies et al.: Detection of hypotension during Caesarean section with continuous non-invasive arterial pressure device or intermittent oscillometric arterial pressure measurement. Br J Anaesth. 2012 Sep;109(3):413-9.

[3] M. Hamilton et al.: A systematic review and meta-analysis on the use of preemptive hemodynamic intervention to improve postoperative outcomes in moderate and high-risk surgical patients. Anesth Analg 112(6), 1392-402. 2011

[4] BP. Imholz et al.: Effects of vasoconstriction upon the measurement of finger arterial pressure. J Hypertens, 10(9), 979-84. 1992

[5] R. Raamat et al.: Different responses of Finapres and the oscillometric finger blood pressure monitor during intensive vasomotion. $J$ Med Eng Technol, 24(3), 95-101. 2000

[6] K. Jagomägi et al.: Effect of altering vasoactivity on the measurement of finger blood pressure. Blood Pres Mon, 6(1), 33-40. 2001

[7] J. Peňáz: Photoelectric Measurement of blood pressure, volume and flow in the finger. Digest of the 10th international conference on medical and biological engineering - Dresden (1973).

[8] DB. Wax et al.: Invasive and concomitant noninvasive intraoperative blood pressure monitoring: observed differences in measurements and associated therapeutic interventions. Anesthesiology, $2011 ; 115(5), 973-8$.

[9] J Fortin, R. Grüllenberger: US 2011/0105918, May $5^{\text {th }} 2011$

[10]RD. Boehmer: Continuous, real-time, noninvasive monitor of blood pressure: Penaz methodology applied to the finger. J Clin Monit. 1987 Oct;3(4):282-7.

[11]J. Fortin et al.: Continuous non-invasive blood pressure monitoring using concentrically interlocking control loops. Comput Biol Med 36 941-957, 2006

[12] AM. Jagadeesh et al.: A comparison of a continuous noninvasive arterial pressure (CNAP) monitor with an invasive arterial blood pressure monitor in the cardiac surgical ICU. Ann Card Anaesth, 15(3), 180-4. 2012

[13]M. Neuner et al.: Clinical validation of a continuous non-invasive haemodynamic monitor (CNAP 500) during general anaesthesia. Brit J Anaesth, 2012 Apr;108(4):581-5.

[14]M. Biais et al.: Continuous non-invasive arterial pressure measurement: evaluation of CNAP device during vascular surgery. $\mathrm{Ann} F r$ Anesth, 29(7-8), 530-5. 2010

[15]C. Ilies et al.: Investigation of the agreement of a continuous noninvasive arterial pressure device in comparison with invasive radial artery measurement. Br J Anaesth. 2012 Feb;108(2):202-10

[16]C. Jeleazcov et al: Precision and accuracy of a new device (CNAP) for continuous non-invasive arterial pressure monitoring: assessment during general anaesthesia. $B r \quad J$ Anaesth. 2010 Sep;105(3):264-72

[17]X. Monnet et al. Prediction of fluid responsiveness by a continuous non-invasive assessment of arterial pressure in critically ill patients: comparison with four other dynamic indices. Br J Anaesth. 2012 Sep;109(3):330-8

[18]M. Biais et al.: The ability of pulse pressure variations obtained with CNAP device to predict fluid responsiveness in the operating room. Anesth Analg. 2011 Sep;113(3):523-8 